\title{
Unilateral Idiopathic Sensory Trigeminal Nerve Paralysis in a Dog
}

\author{
Maria Luíza de Melo Dias' \& Ana Carolina Mortari²
}

\begin{abstract}
Background: A spayed-female mixed breed dog was presented due to 2 years reduced visual acuity in the left eye. The investigation revealed corneal melanosis, keratoconjunctivis sicca and loss of facial sensation in the same side. The aim of this report is to describe an unilateral idiopathic sensory trigeminal nerve paralysis and keratitis as consequence of corneal innervation loss was established. Unilateral sensory trigeminal nerve paralysis is a very rare lesion in dogs and causes are unclear.

Case: A 5-year-old spayed-female mixed breed dog was presented with a history of two years reduced visual acuity in the left eye. The Schirmer tear test values (without use of topical anesthetic) were 11 and $17 \mathrm{~mm} / \mathrm{min}$ in the left and right eye respectively. Fluorescein tests were negative for both eyes. All postural reactions and spinal reflexes were normal. Cranial nerve evaluation identified symmetrical facial muscles (temporalis, masseter), indicating no muscle atrophy and normal motor nucleus of the fifth nerve. Absent sensation at the upper and lower lips margins, cornea, eyelids (medial and lateral canthi) and sensation in left nasal side were noted. No palpebral reflex was noticed, but there was spontaneous blinking of eyelids, which indicated normal facial nerve function. All other cranial nerve reflexes including menace response, oculocephalic, and gag reflexes were normal. The owner declined further workup, including cerebrospinal fluid analysis and images studies. According to the clinical presentation and neurological findings, presumptive diagnosis of unilateral idiopathic sensory trigeminal nerve paralysis was established. The dog was observed over 18 months without any clinical improvement. The Schirmer tear test showed same values $(12 \mathrm{~mm} / \mathrm{min})$ and the melanosis remained unchanged.

Discussion: Lesions that affected trigeminal nerve, trigeminal ganglion, or trigeminal tract in the pons and medulla caused facial hypoalgesia or analgesia. The causes of trigeminal nerve damage included neoplasia, trauma, fracture of petrous temporal bone and inflammatory polyneuropathies, but the dog present no signals of these diseases. Trigeminal nerve lesions that affected ophthalmic nerve branch will result in loss of corneal sensation and loss of corneal and palpebral reflex as consequence. Paralysis of this branch cause degenerative changes in the cornea, as neurotrophic keratitis that result in edema and epitelial erosion. The Schirmer tear is the standard test to quantify aqueous tear production and the result (11 $\mathrm{mm} / \mathrm{min}$ ) in dog's left eye is considered early or subclinical keratoconjunctivis sicca (KCS) sign. The pigmentation results from melanocytic cells migration from the limbal or perilimbal tissues. The keratitis can be active when followed by corneal vascularization, stromal inflammatory cell infiltration and granulation tissue formation. The etiology for idiopathic disease is unknown and isolated sensory trigeminal neuropathy have never been described in dogs. There is no definitive treatment and corticosteroid therapy did not appear to alter the course of the disease in dogs with idiopathic trigeminal neuritis therefore supportive treatment is indicated. The paralysis of trigeminal sensory ophthalmic and maxillary branches can result in corneal alterations.
\end{abstract}

Keywords: dog, canine, trigeminal neuropathy, corneal disease. 


\section{INTRODUCTION}

The trigeminal nerve contains both motor and sensory components and the sensory part is the larger. In trigeminal canal on the petrosal part of the temporal bone is located the trigeminal ganglion that contains sensory cell bodies of the general somatic afferent axons found in all three branches of this nerve (ophthalmic, maxillary, mandibular). Ophthalmic and maxillary branches had sensory fibers nerve and the mandibular branch contains both motor and sensory fibers [3].

In trigeminal neuropathy, one third of dogs have involvement of general somatic afferent neurons in all three nerve branches causing facial loss sensation [2,7]. Lesions in the ophthalmic nerve branch of trigeminal nerve may result in understood corneal degenerative changes [2].

Unilateral sensory trigeminal nerve paralysis is a very rare lesion in dogs and causes are unclear [1]. To the authors' knowledge there have been no previously published reports of isolated unilateral sensory trigeminal nerve paralysis.

\section{CASE}

A 5-year-old spayed-female mixed breed dog was presented at the Ophthalmology Service in the Veterinary Teaching Hospital with two years reduced visual acuity in the left eye. According to the owner, signs were present when the dog was adopted and no treatment was initiated previously. On physical examination, no abnormalities were noted. Moderate hyperemia, neovascularization and a great area of melanosis were noted on ophthalmic examination of the left eye. The right eye was normal. The Schirmer tear test values (without use of topical anesthetic) were 11 and $17 \mathrm{~mm} / \mathrm{min}$ in the left and right eye respectively, indicating slight reduction in left eye. Fluorescein tests were negative for both eyes.

The dog was referred to the neurologic service due to absence of facial sensation. Gait evaluation was normal with no ataxia or weakness. All postural reactions and spinal reflexes were normal and no pain was elicited on spinal palpation. Cranial nerve evaluation identified symmetrical facial muscles (temporalis, masseter), indicating no muscle atrophy and normal motor nucleus of the fifth nerve (Figure 1A). Absent sensation at the upper and lower lips margins, cornea (Figure 1B), eyelids (medial and lateral canthi) and sensation in left nasal (Figure 1C \& D) side were noted. No palpebral reflex was noticed, but there was spontaneous blinking of eyelids, which indicated normal facial nerve function. All other cranial nerve reflexes including menace response, oculocephalic, and gag reflexes were normal.

A complete blood count, biochemistry profile (renal and liver function), triglycerides, cholesterol, glucose, albumin, thyroid function tests were performed and no abnormalities were detected. Thyroid function tests were within normal limits. Thoracic radiographs and abdominal ultrasound examination were unremarkable. The owner declined further workup, including cerebrospinal fluid analysis and images studies. Acupuncture was recommended but the owner also declined it.

According to the clinical presentation and neurological findings, presumptive diagnosis of unilateral idiopathic sensory trigeminal nerve paralysis was established. The dog was observed over 18 months without any clinical improvement. The Schirmer tear test showed same values $(12 \mathrm{~mm} / \mathrm{min})$ and the melanosis remained unchanged.

\section{DISCUSSION}

Sensory fibers of mandibular branch of trigeminal nerve innervate buccal cavity, tongue, tooth and mandible skin, caudal buccal region, and craniolateral pinna. Maxillary branch innervates lower eyelid, nasal mucosa, upper tooth and lip, and nose. Ophthalmic branch innervates eyelids, eyeball, nasal mucosa, and nose skin [3].

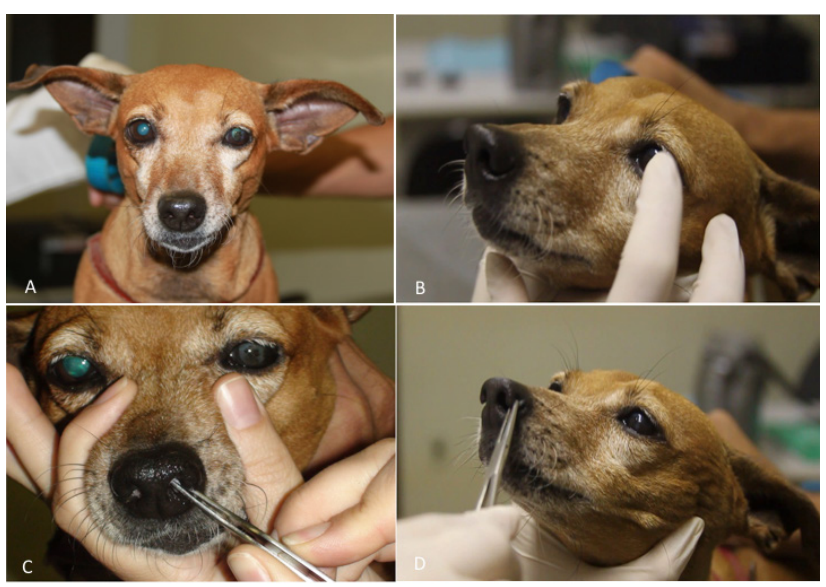

Figure 1. Neurologic examination of the dog. A- The left eye showed neovascularization and central melanosis. The right eye was grossly normal. The temporalis and masseter muscles were symmetrical, indicating no muscle atrophy. B- The left eye showed absence of sensation in the cornea. C- The neurological examination showed absence of sensation in the nasal mucosa (front). D- The neurological examination showed absence of sensation in the nasal mucosa (lateral). 
Lesions that affected trigeminal nerve, trigeminal ganglion, or trigeminal tract in the pons and medulla caused facial hypoalgesia or analgesia [2,9,12]. If vision and the facial nerve are not involved, menace response should still be normal [9]. The lesion of trigeminal nerve has an inflammatory pattern with predominantly primary desmyelination caused by direct or indirect infection $[2,11]$. The disease affecting motor and sensory nerve branches has no breed prevalence but Golden Retrievers were overrepresented in one study. In the present case, the signs were restricted to facial sensory loss (corneal, nasal, lip, canthi of eye) in the left side with secondary ophthalmic alterations, indicating restrict involvement of trigeminal sensory nerve component (mandibular, maxillary and ophthalmic branches). A pure sensory neuropathy of trigeminal nerve is rare and causes are unclear. A case of isolated sensory trigeminal neuropathy has been reported in two-year-old female collie presenting hypersalivation, coughing, dysphagia and bilateral facial sensory loss over the distribution of trigeminal nerve. Trigeminal motor function was preserved and no cause could be found [1].

A presumptive diagnosis to trigeminal neuropathy can be made based on history and neurological exam findings [7]. In cases where trigeminal nerve motor component is affected, most dogs recover spontaneously in 3 to 4 weeks [2,7] but there is no recovery description for sensory disease.

The causes of trigeminal nerve damage included neoplasia, trauma, fracture of petrous temporal bone and inflammatory polyneuropathies [9] but the dog present no signals of these diseases. Infection, immune-mediated and allergic causes for the condition also have been proposed $[2,7]$ although no evidence to support these hypotheses was presented. The dog was tested for hypothyroidism and the result was negative as observed in another study [7].

Trigeminal nerve lesions that affected ophthalmic nerve branch will result in loss of corneal sensation and loss of corneal and palpebral reflex as consequence. Paralysis of this branch cause degenerative changes in the cornea [2], as neurotrophic keratitis that result in edema and epithelial erosion $[9,10]$. The negative fluorescein tests indicate no ulcerative lesion in the cornea. Corneal pigmentation is most commonly associated with chronic inflammation like as chronic superficial keratitis, keratoconjuntivitis sicca and chronic ulcerative keratitis. Congenital form is rare in dogs. The pigmentation results from melanocytic cells migration from the limbal or per- ilimbal tissues. The keratitis can be active when followed by corneal vascularization, stromal inflammatory cell infiltration and granulation tissue formation [6]. In this case, the dog presented corneal sensory loss, extensive corneal pigmentation and corneal vascularization indicating previous corneal inflammation that can be associated to trigeminal nerve sensory lesion. The relation between the corneal cellular metabolism and its sensory nerve supply is poorly understood [2]. No other ophthalmic diseases evidence was found.

The Schirmer tear is the standard test to quantify aqueous tear production and the result $(11 \mathrm{~mm} / \mathrm{min})$ in dog's left eye is considered early or subclinical keratoconjunctivis sicca (KCS) sign [4,6]. Ophthalmic nerve divides into three branches: frontal, lacrimal and nasociliary nerve. The maxillary branch and lacrimal nerve contain postganglionic parasympathetic fibers that innervate lacrimal gland [3]. The ophthalmic branch mediates reflex tear production in response to stimulation of the cornea and nasal mucosa (sensory innervation) and, occasionally, the production decreases when this function is lost in the cranial nerve V lesion, causing KCS [6,13]. For this reason, although basal tear secretion should be normal, reflex tear production in response to stimulation of the cornea is lost [9]. In chronic corneal disease, melanin pigment granules can be noted throughout the corneal epithelium that becomes extensively vascularized [6].

There is no definitive treatment $[2,11]$ and corticosteroid therapy did not appear to alter the course of the disease in dogs with idiopathic trigeminal neuritis therefore supportive treatment is indicated. Acupuncture had been indicated to treat pain, paralysis (both flaccid and spastic) and muscle wasting due to beneficial effects on microcirculation, inflammation and nerve damage $[5,8]$ and it was recommended in this case but the owner declined it.

The etiology for idiopathic disease is unknown and isolated sensory trigeminal neuropathy have never been described in dogs. One published report, histopathology of trigeminal neuritis revealed demyelination and non-suppurative neuritis of all portions of trigeminal nerve and ganglion [2].

In conclusion, unilateral sensory trigeminal nerve paralysis is a rare condition characterized by loss of corneal and facial sensation. The paralysis of trigeminal sensory ophthalmic and maxillary branches can result in corneal alterations.

Declaration of interest. The authors report no conflicts of interest. The authors alone are responsible for the content and writing of the paper. 


\section{REFERENCES}

1 Carmichael S. \& Griffiths I.R. 1981. Case of isolated sensory trigeminal neuropathy in a dog. Veterinary Record. 109(13): 280-282.

2 De Lahunta A., Glass E. \& Kent M. 2015. Lower Motor Neuron: Spinal Nerve, General Somatic Efferent System. In: De Lahunta A., Glass E. \& Kent M. (Eds). Veterinary Neuroanatomy and Clinical Neurology. 4th edn. Philadelphia: Elsevier-Saunders, pp.102-161.

3 Evans H.E. \& De Lahunta A. 2013. Cranial nerves. In: Evans H.E. \& De Lahunta A. (Eds). Miller's anatomy of the dog. 4th edn. St. Louis: Elsevier-Saunders, pp.708-729.

4 Hamor R.E., Roberts S.M., Severin G.A. \& Chavkin M.J. 2000. Evaluation of results for Schirmer tear tests conducted with or without application of a topical anesthetic in clinical normal dogs of 5 breeds. American Journal of Veterinary Research. 61(11): 1422-1425.

5 Jeong S.M., Kim H.Y., Lee C.H., Kweon O.K. \& Nam T.C. 2001. Use of acupunture for treatment of idiopathic facial nerve paralysis in a dog. Veterinary Record. 148(20): 632-633.

6 Ledbetter E.C. \& Gilger B.C. 2013. Diseases and surgery of the canine cornea and sclera. In: Gellatt K., Gilger B.C. \& Kern T.J. (Eds). Veterinary Ophthalmology. 4th edn. Ames: Willey-Blackwell, pp.976-1049.

7 Mayhew P.D., Bush W.W. \& Glass E.N. 2002. Trigeminal neuropathy in dogs: A retrospective study of 29 cases (1991-2000). Journal of the American Animal Hospital Association. 38(3): 262-270.

8 Mayor D.F. 2007. Electroacupuncture: An introduction and its use for peripheral facial paralysis. Journal of Chinese Medicine. 84: 52-70.

9 Penderis J. 2004. Disorders of eyes and vision. In: Platt S.R. \& Olby N.J. (Eds). BSAVA Manual of canine and feline neurology. 3rd edn. Quedgeley: BSAVA, pp.133-154.

10 Porter B., Schatzberg S., Mcdonough S., Mertens D. \& De Lahunta A. 2002. Ganglioradiculitis (sensory neuropathy) in a dog: clinical, morphologic and immunohstochemical findings. Veterinary Pathology. 39(5): 598-602.

11 Powell A.K. 1991. Idiopathic trigeminal neuritis in a dog. Canadian Veterinary Journal. 32(5): 265.

12 Thomson C. \& Hanh C. 2012. Cranial nerves. In: Thomson C. \& Hanh C. (Eds). Veterinary Neuroanatomy. St. Louis: Saunders Elsevier, pp.91-108.

13 Uemura E.E. 2015. Anatomy of the Canine Brain In: Uemura E.E. (Ed). Fundamentals of canine neuroanatomy and neurophysiology. Ames: Willey-Blackwell, pp.38-84. 